\title{
INCERTIDUMBRE EN LA PREDICCIÓN DEL VIENTO MEDIANTE UN SISTEMA DE PREDICCIÓN POR CONJUNTOS: CUANTIFICACIÓN Y ANÁLISIS
}

\author{
Mariano Sastre $^{(1)}$, Sergio Fernández-González ${ }^{(2)}$ Francisco Valero $^{(1)}$, \\ María Luisa Martín ${ }^{(3)}$. \\ ${ }^{(1)}$ Departamento de Física de la Tierra y Astrofísica, Facultad de Ciencias Físicas, Universidad Complutense de \\ Madrid, Plaza Ciencias 1, Madrid, España, msastrem@ucm.es \\ (2) Agencia Estatal de Meteorología (AEMET), Leonardo Prieto Castro 8, Madrid, España \\ (3) Departamento de Matemática Aplicada, Escuela de Ingeniería Informática, Universidad de Valladolid, Plaza de \\ la Universidad 1, Segovia, España
}

\section{Resumen}

A partir de un sistema de predicción por conjuntos generado con un modelo meteorológico mesoescalar, y mediante un índice basado en la dispersión de los miembros del conjunto, se evalúa la incertidumbre asociada en la predicción de la velocidad del viento a corto plazo en terreno complejo. Se analizan los resultados diferenciando entre distintos casos, según la dirección del viento y si se rebasa un determinado umbral de velocidad del mismo, así como considerando distintas posibilidades de condiciones de estabilidad atmosférica. Así mismo, se separa la contribución a la incertidumbre correspondiente a las parametrizaciones físicas del modelo, por una parte, y a las condiciones iniciales y de contorno por otra. En la mayor parte de los casos la contribución de las condiciones iniciales y de contorno es menor, pero debe ser especialmente tenida en cuenta en determinadas áreas y circunstancias meteorológicas.

\section{Introducción}

El indudable desarrollo de la energía eólica actual la ha llevado a que, dentro de las renovables, sea ya la segunda a nivel mundial en términos de capacidad instalada (Santos et al., 2015). Sin embargo, su producción conlleva problemas de diversa índole, tales como la necesidad de un pronóstico del viento muy preciso para su adecuada integración en el sistema de la red eléctrica (Najafi et al., 2016). Además, también deben tenerse en cuenta los efectos estructurales de la cizalladura y las rachas fuertes de viento sobre los aerogeneradores (Worsnop et al., 2017). Por ello, disponer de una predicción ajustada es realmente crucial, tanto para minimizar los daños potenciales durante los episodios de vientos muy fuertes, como para el diseño óptimo de los parques eólicos.

Dado que los modelos atmosféricos globales suelen subestimar la velocidad del viento, especialmente en terreno complejo, el uso de modelos mesoescalares está muy extendido en este ámbito. Sin embargo, incluso con modelos de alta resolución de este tipo también hay incertidumbre en el pronóstico de la velocidad del viento, que es muy conveniente estimar. Una solución para hacer esta estimación es desarrollar un sistema de predicción por conjuntos (EPS, por sus siglas en inglés) compuesto por varios miembros (Slingo and Palmer, 2011). Este es el método que se utiliza en este trabajo, construyendo el EPS a partir de simulaciones con un mismo modelo al que se le ha introducido alguna variación en la configuración de las simulaciones, como pueden ser las condicio- 
nes iniciales, o el esquema de capa límite utilizado, entre otros. Una vez se cuenta con el EPS, el objetivo de este estudio es cuantificar la incertidumbre de la predicción por este método, utilizando para ello un índice de dispersión aplicado a los resultados del EPS. En particular, se sigue la metodología y el índice de dispersión definido por FernándezGonzález et al. (2017a). También se analiza la incertidumbre en distintos escenarios: dependiendo de la velocidad y dirección del viento, y de las condiciones de estabilidad atmosférica en la parte baja de la atmósfera. Por último, se estudia la distribución geográfica de la incertidumbre de la predicción y la contribución a la misma de diferentes características del modelo.

\section{Simulaciones}

El modelo hidrostático mesoescalar Weather Research and Forecasting (WRF) (Skamarock and Klemp, 2008) es una herramienta muy popular entre la comunidad científica meteorológica. Durante los últimos años se ha empleado satisfactoriamente para estudiar fenómenos atmosféricos de diversa índole; sirvan como ejemplos las brisas marinas (Arrillaga et al., 2016), episodios de nieblas radiativas (Román-Cascón et al., 2016), tormentas severas (Gascón et al., 2015), engelamiento en aeronaves (FernándezGonzález et al., 2014), o la transición vespertina de la capa límite atmosférica (Sastre et al., 2012; 2015). En este trabajo se realizan simulaciones con la versión 3.6.1 de WRF, utilizando condiciones iniciales y de contorno del reanálisis de ERA-Interim (ERA) y de NCEP-GFS. Además, dos esquemas de uso de suelo-superficie, y dos parametrizaciones de capa límite, de capa superficial, y de radiación atmosférica fueron utilizados. En concreto, se han utilizado los mismos esquemas y parametrizaciones que en el trabajo de Fernández-González et al. (2017b).

Combinando estas posibilidades, se obtuvo finalmente un total de 16 simulaciones. Todas ellas tuvieron como característica común el hecho de usar 4 dominios anidados

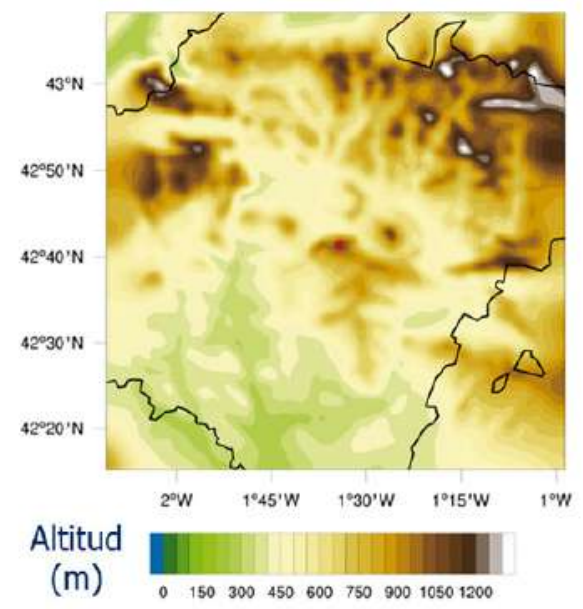
de $100 \times 100$ puntos, centrados en un punto del norte de la península ibérica. Las resoluciones espaciales de los dominios fueron de 27, 9, 3 y $1 \mathrm{~km}$, cubriendo el dominio 1 gran parte de Europa Occidental, mientras que el dominio 4 abarcaba principalmente la Comunidad Foral de Navarra (España). El estudio se focaliza en este último dominio (Figura 1). Se trata de una zona

Figura 1. Orografía del área de estudio (dominio 4 de las simulaciones). La línea negra continua indica el límite geográfico de la Comunidad Foral de Navarra. El punto rojo corresponde a uno de los parques eólicos de la zona. 
de terreno complejo, en la cual la presencia de parques eólicos puede ser utilizada para validar los resultados con observaciones. En cuanto a la resolución vertical, se usaron 61 niveles sigma con una mayor proporción de los mismos cerca de la superficie (21 de ellos entre el suelo y $1000 \mathrm{~m}$, de los cuales ocho estaban en los primeros $120 \mathrm{~m}$ ). Todas las simulaciones se inicializaron a las $00 \mathrm{UTC}$, considerando las primeras $12 \mathrm{horas}$ como spin-up, y tomando las siguientes 24 horas para el análisis. Por tanto, la predicción que se evalúa es a corto plazo. En total se simulan 15 días individualmente, pertenecientes al mes de mayo de 2015.

\section{Cálculo y análisis de la incertidumbre}

Una vez generado el EPS con las simulaciones descritas en el apartado anterior, se calcula el índice de dispersión definido por Fernández-González et al. (2017a), que será indicador de la incertidumbre de la predicción de viento. Para analizar y cuantificar en detalle esta incertidumbre, se clasifican las simulaciones realizadas según la intensidad del viento, la dirección del mismo y la estructura térmica atmosférica:

- Intensidad del viento. Se utiliza como criterio el valor de la media del EPS en el nivel de $90 \mathrm{~m}$ sobre el nivel del suelo, tomando como umbral el promedio calculado en todo el dominio 4 para los días simulados, que es de $7 \mathrm{~m} \mathrm{~s}^{-1}$ : valores superiores se consideran viento fuerte y valores inferiores viento débil.

- Dirección del viento. Análogamente, se diferencia entre viento del norte si la dirección del promedio del EPS da valores de $0^{\circ} \pm 60^{\circ}$, y viento del sur para los casos cuya dirección sea $180^{\circ} \pm 60^{\circ}$. El nivel que se toma también es el de $90 \mathrm{~m}$ sobre el nivel del suelo.

- Estructura térmica atmosférica. Se consideran cuatro posibilidades, según los valores de temperatura y temperatura potencial $(\theta)$ en los niveles de $38 \mathrm{~m}$ y $97 \mathrm{~m}$ sobre el nivel del suelo: inestable $(\Delta \theta / \Delta \mathrm{z}<0)$, neutral $(\Delta \theta / \Delta \mathrm{z}=0)$, estable $(\Delta \theta / \Delta \mathrm{z}>0)$, e inversión térmica.

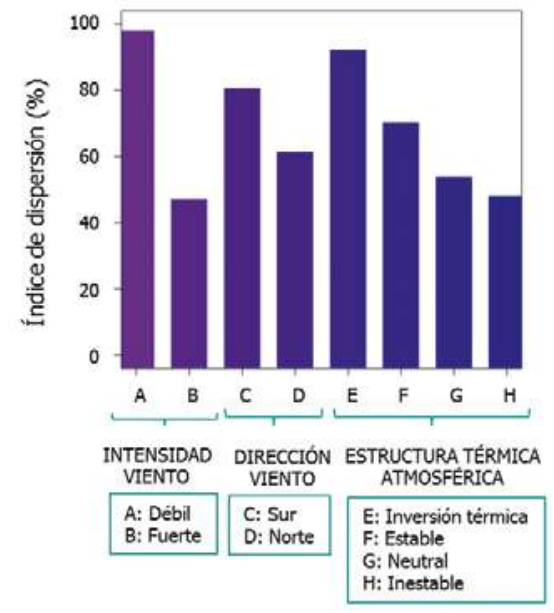

Con estos criterios, calculando el promedio espacial considerando el nivel de $90 \mathrm{~m}$, se observan diferencias significativas en la capacidad de predicción del viento, según los distintos casos (Figura 2).

Figura 2. Índice de dispersión (incertidumbre) según distintos escenarios de intensidad y dirección de viento, y de la estructura térmica atmosférica.

Por otra parte, analizando este mismo índice en los diferentes niveles verticales que proporciona el modelo (Figura 3), se aprecia cómo generalmente la incertidumbre disminuye con la altura con algunas excepciones de eventos de viento débil y condiciones de inversión térmica. 


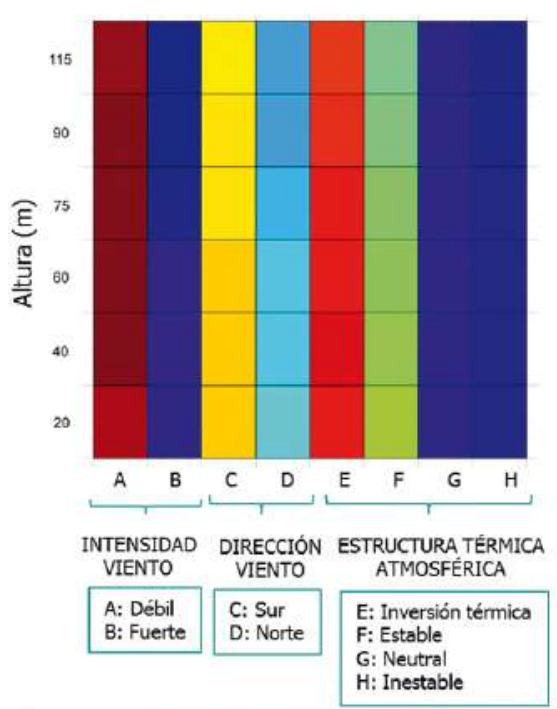

Indice de dispersión

(\%)
Figura 3. Promedio del índice de dispersión para los días de estudio simulados a varios niveles verticales, según distintos escenarios de intensidad y dirección de viento, y de condiciones de estabilidad atmosférica.

Finalmente se realiza una representación a nivel espacial, tanto de la incertidumbre total en el dominio 4, como de las contribuciones a la incertidumbre de las distintas fuentes de la misma dentro del EPS. En este caso se han separado estas contribuciones en dos categorías: las correspondientes a las parametrizaciones físicas del modelo y las que hacen referencia a las condiciones iniciales y de contorno del mismo. Los resultados muestran que, globalmente, para las predicciones a corto plazo la incertidumbre debida a las parametrizaciones físicas es mayor $(63,5 \%)$. Esto es lo que ocurre, por ejemplo, en el caso de las situaciones de viento débil (Figura 4).
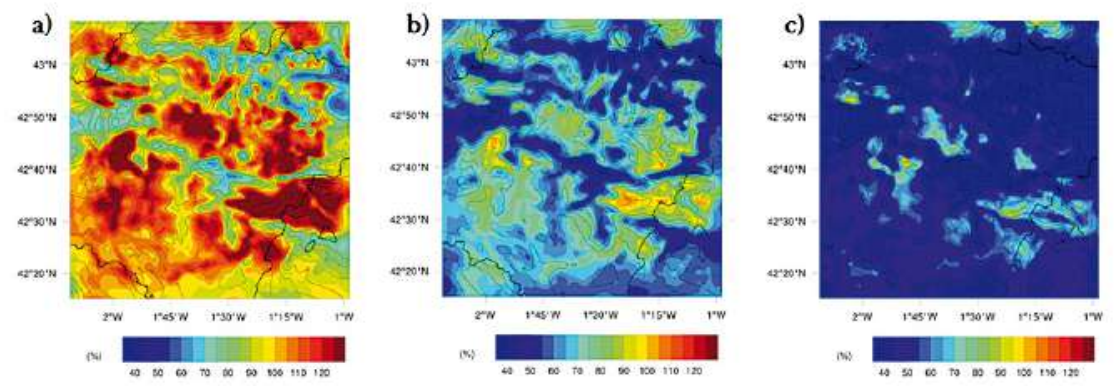

Figura 4. Distribución espacial promedio del índice de dispersión para los casos de viento débil: a) incertidumbre total; b) contribución a la incertidumbre debida a las parametrizaciones físicas; c) contribución a la incertidumbre debida a las condiciones iniciales y de contorno.

Sin embargo, también es necesario tener en cuenta la incertidumbre asociada a las condiciones iniciales, no solo por su importancia cuantitativa a nivel global $(36,5 \%)$, sino también por su comportamiento durante ciertas condiciones meteorológicas o climáticas. En particular, en algunas zonas concretas del área de estudio se aprecia que la contribución a la incertidumbre de las condiciones iniciales y de contorno es la fuente principal de dicha incertidumbre para los casos de viento del sur (Figura 5). 

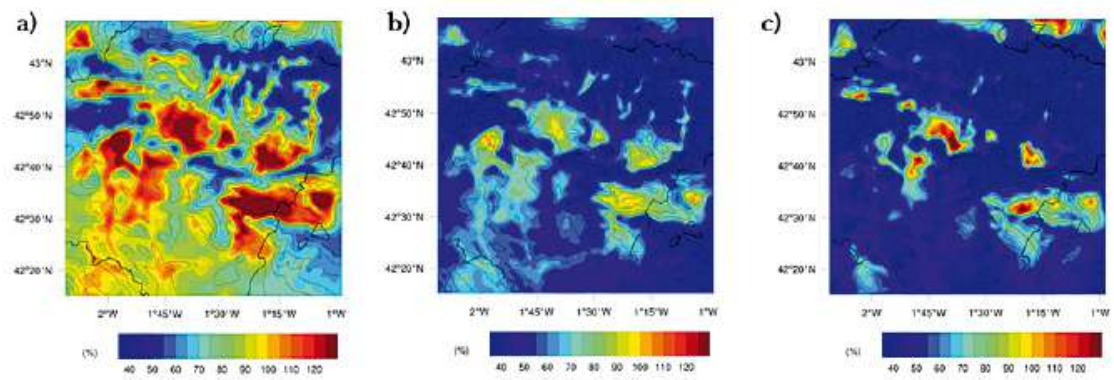

Figura 5. Distribución espacial promedio del índice de dispersión para los casos de viento del sur: a) incertidumbre total; b) contribución a la incertidumbre debida a las parametrizaciones físicas; c) contribución a la incertidumbre debida a las condiciones iniciales y de contorno.

Un efecto similar se aprecia en el caso de tener una estructura atmosférica de inversión térmica (Figura 6). De hecho, el patrón geográfico de contribución de las condiciones iniciales y de contorno a la incertidumbre total tiene grandes similitudes con el análogo para los casos de viento del sur, aunque los respectivos patrones de incertidumbre total no guarden tanta semejanza. Esto sugiere que hay ciertas áreas geográficas que, debido a su complejidad, son más sensibles a las condiciones iniciales y de contorno a la hora de obtener una predicción de viento fiable.
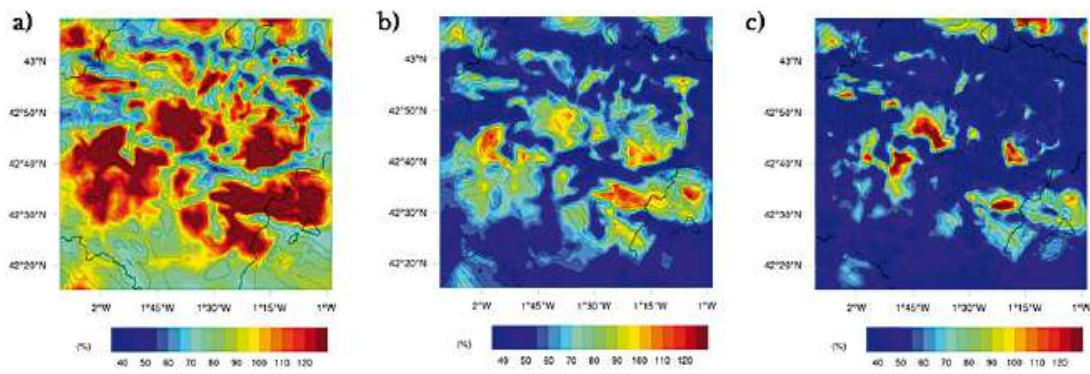

Figura 6. Distribución espacial promedio del índice de dispersión para los casos de inversión térmica: a) incertidumbre total; b) contribución a la incertidumbre debida a las parametrizaciones físicas; c) contribución a la incertidumbre debida a las condiciones iniciales y de contorno.

\section{Conclusiones}

En este trabajo se ha mostrado cómo las áreas con los vientos más intensos presentan mayoritariamente una mejor predictibilidad del mismo. Además, en los perfiles verticales se observa que la incertidumbre disminuye con la altura en la mayor parte de situaciones atmosféricas. Estos dos hechos suponen una gran ventaja en el contexto de la predicción del recurso eólico. 
En cuanto al análisis según la estructura térmica atmosférica, la incertidumbre más elevada aparece durante las condiciones de inversión térmica.

Por último, separar la contribución a la incertidumbre según si es debida a las condiciones iniciales y de contorno o las parametrizaciones físicas permite ver las diferencias a nivel geográfico. Globalmente se ha observado que la incertidumbre debida a condiciones iniciales y de contorno suele ser menor, pero no debe descartarse, ya que puede llegar a ser muy relevante en ciertas situaciones, principalmente con viento del sur y episodios de inversión térmica en determinadas zonas de nuestra área de estudio.

Los resultados aquí presentados pueden ser de utilidad para escoger futuros emplazamientos de parques eólicos, considerando la incertidumbre de la predicción como un factor de gran relevancia. La extensión natural de este trabajo consistiría en aplicar la misma metodología a áreas más amplias y con un número de casos simulados mayor, de modo que las conclusiones puedan ser validadas en un espectro temporal y espacial mayor.

\section{Referencias}

- Arrillaga, J. A., C. Yagüe, M. Sastre, and C. Román-Cascón, 2016: A characterisation of sea-breeze events in the eastern Cantabrian coast (Spain) from observational data and WRF simulations, Atmos. Res., 181, 265-280.

- Fernández-González, S., J. L. Sánchez, E. Gascón, L. López, E. García-Ortega, and A. Merino, 2014: Weather Features Associated with Aircraft Icing Conditions: A Case Study, The Scientific World Journal, 2014, Article ID 279063, 18 páginas, doi:10.1155/2014/279063.

- Fernández-González, S, M.L. Martín, A. Merino, J.L. Sánchez, and F. Valero, 2017a: Uncertainty quantification and predictability of wind speed over the Iberian Peninsula. J. Geophys. Res. Atmos., 122, 3877-3890.

- Fernández-González, S., M. L. Martín, E. García-Ortega, A. Merino, J. Lorenzana, J. L. Sánchez, F. Valero, and J. Sanz Rodrigo, 2017b, Sensitivity analysis of WRF model: wind-resource assessment for complex terrain. J. Appl. Meteor. Climatol., doi:10.1175/JAMC-D-17-0121.1

- Najafi, A., H. Falaghi, J. Contreras, and M. Ramezani, 2016: Medium-term energy hub management subject to electricity price and wind uncertainty. Appl. Energy, 168, 418-433.

- Román-Cascón, C., C. Yagüe, G.-J. Steeneveld, M. Sastre, J. A. Arrillaga, and G. Maqueda, 2016: Estimating fog-top height through near-surface micrometeorological measurements. Atmos. Res., 170, 76-86.

- Santos, J.A., C. Rochinha, M.L.R. Liberato, M. Reyers, and J. Pinto, 2015: Projected changes in wind energy potentials over Iberia. Renewable Energy, 75, 68-80.

- Sastre, M., C. Yagüe, C. Román-Cascón, G. Maqueda, F. Salamanca, and S. Viana, 2012: Evening transitions of the atmospheric boundary layer: characterization, case studies and WRF simulations, Advances in Science and Research, 8, 39-44.

- Sastre, M., C. Yagüe, C. Román-Cascón, and G. Maqueda, 2015: Atmospheric boundary-layer evening transitions: a comparison between two different experimental sites, Bound. Layer Meteorol. 157, 375-399.

- Skamarock, W.C., and J.B. Klemp, 2008: A time-split nonhydrostatic atmospheric model for weather research and forecasting applications. J. Comput. Phys., 227, 3465-3485. 
- Slingo, J., and T. Palmer, 2011: Uncertainty in weather and climate prediction. Philos. Trans. Roy. Soc. London, A369, 4751-4767.

- Worsnop, R.P., J.K. Lundquist, G.H. Bryan, R. Damiani, and W. Musial, 2017: Gusts and shear within hurricane eyewalls can exceed offshore wind turbine design standards. Geophys. Res. Lett., 44, 6413-6420.

\section{Agradecimientos}

Este trabajo ha sido financiado parcialmente por los proyectos de investigación PCIN-2014-013-C07-04, PCIN2016-080 (UE ERA-NET Plus, Proyecto NEWA), CGL2016-78702-C2-1-R y CGL2016-78702-C2-2-R, CGL2016-81828-REDT, así como por el Instituto de Matemática Interdisciplinar (IMI) de la Universidad Complutense de Madrid. 